\title{
Performance of Density Functionals for the Calculation of Gold Clusters
}

\author{
Seol Ryu, Hyun Won Lee, ${ }^{\dagger}$ and Young-Kyu Han ${ }^{\dagger, *}$ \\ Department of Chemistry, Chosun University, Gwangju 501-759, Korea \\ †Division of Materials Science, Korea Basic Science Institute (KBSI), Daejeon 305-333, Korea. *E-mail: ykhan@kbsi.re.kr \\ Received May 24, 2011, Accepted June 20, 2011
}

Key Words : Density functional calculations, Gold, Heavy element, Metal cluster

Clusters and nanoparticles of gold have received considerable attention during the past decade..$^{1-4}$ The exceptional catalytic properties of small gold aggregates ${ }^{5,6}$ have motivated research aimed at providing insights into the molecular origins of this unexpected reactivity. ${ }^{7}$ The experimental observations have stimulated many theoretical studies of the electronic, structural, and chemical properties of gold clusters. $^{8-13}$

Since it is not practical to calculate gold clusters using high-level ab initio correlation methods, density functional theory (DFT)-based approaches have usually been employed in such calculations, but it is not clear which functionals provide the best performance. Herein we report the results of calculations on the structure and stability of $\mathrm{Au}_{2}$ and $\mathrm{Au}_{8}$, as a model study, using various density functionals. To the best of our knowledge, there has been no previous systematic study for DFT performance on the structure and stability of gold clusters. Although the performance of density functionals is widely known for light element systems, ${ }^{14-16}$ certain functionals successful in light element chemistry may not work effectively in heavy element chemistry. This is due to the strikingly different bonding nature of heavy element systems from their light element analogues. ${ }^{17}$ Thus, the results of this work may be useful for future work in choosing the most appropriate density functional for gold clusters when performing DFT calculations.

Kohn-Sham DFT calculations were performed with 16 different exchange correlation functionals, namely, the local density approximation (LDA:SVWN) ${ }^{18}$ the generalized gradient approximation (GGA:BLYP, BP86, BPW91, PW91, PBE, HCTH, tHCTH, LC-BPW91, LC-PW91 and LCPBE) ${ }^{18}$ and the hybrid GGA functionals (B3LYP, B3PW91, mPW1PW91, PBE0 and X3LYP). ${ }^{18}$ We used the relativistic effective core potentials derived by Stevens et al. and valence basis sets ${ }^{19}$ employed in previous works. ${ }^{9,10}$ All the calculations were carried out using the program package GAUSSIAN 09. ${ }^{18}$

$\mathrm{Au}_{2}$ Cluster. We compare the calculated spectroscopic constants, bond length $\left(R_{\mathrm{e}}\right)$, vibrational frequency $\left(\omega_{\mathrm{e}}\right)$, and dissociation energy $\left(D_{e}\right)$ with the experimental data ${ }^{20}$ in Table 1. Overall, the LDA and several GGA functionals provide good performance for bond length (and vibrational frequency) and dissociation energy, respectively. The use of hybrid GGA functionals, i.e., the inclusion of Hartree-Fock exchange, does not improve the pure GGA results. These observations are in contrast to the known performance of functionals in light element chemistry: in general, LDA < GGA $<$ hybrid GGA. It was reported, for instance, that the performance of the pure BP86 functional is very poor for light element systems, ${ }^{21,22}$ but its performance is observed to be one of the most effective for $\mathrm{Au}_{2}$. It is evident from our calculations that any one functional could not provide reliable spectroscopic constants of $\mathrm{Au}_{2}$. The poor performance of hybrid GGA functionals may be partly ascribed to the functional parameterization optimal to light-element systems only. Intriguingly, the long range correction to the GGA functionals (LC-GGA) improves the performance for bond lengths.

Aus Cluster. There are no experimental data for the structures and energies of $\mathrm{Au}_{8} . \mathrm{Han}^{10}$ reported the relative

Table 1. Bond lengths, vibrational frequencies, and dissociation energies of $\mathrm{Au}_{2}$

\begin{tabular}{|c|c|c|c|}
\hline & $\mathrm{R}_{\mathrm{e}}(\AA)$ & $\omega_{\mathrm{e}}\left(\mathrm{cm}^{-1}\right)$ & $\mathrm{D}_{\mathrm{e}}(\mathrm{eV})$ \\
\hline \multicolumn{4}{|c|}{ Local density approximation } \\
\hline SVWN & 2.454 & 195.9 & 3.04 \\
\hline \multicolumn{4}{|c|}{ Generalized gradient approximation } \\
\hline BLYP & 2.556 & 164.7 & 2.17 \\
\hline BP86 & 2.519 & 174.8 & 2.33 \\
\hline BPW91 & 2.522 & 173.5 & 2.23 \\
\hline PW91 & 2.518 & 175.2 & 2.38 \\
\hline PBE & 2.519 & 174.2 & 2.36 \\
\hline HCTH & 2.537 & 163.4 & 2.08 \\
\hline tHCTH & 2.510 & 173.5 & 2.26 \\
\hline LC-BPW91 & 2.463 & 199.0 & 2.09 \\
\hline LC-PW91 & 2.463 & 199.1 & 2.09 \\
\hline LC-PBE & 2.461 & 199.6 & 2.10 \\
\hline \multicolumn{4}{|l|}{ Hybrid GGA } \\
\hline B3LYP & 2.545 & 169.0 & 2.02 \\
\hline B3PW91 & 2.519 & 175.8 & 2.06 \\
\hline mPW1PW91 & 2.517 & 177.0 & 2.05 \\
\hline PBE0 & 2.515 & 177.1 & 2.10 \\
\hline X3LYP & 2.543 & 169.6 & 2.04 \\
\hline Exp. $^{a}$ & 2.476 & 190.0 & 2.24 \\
\hline
\end{tabular}

${ }^{a}$ Reference [20] 


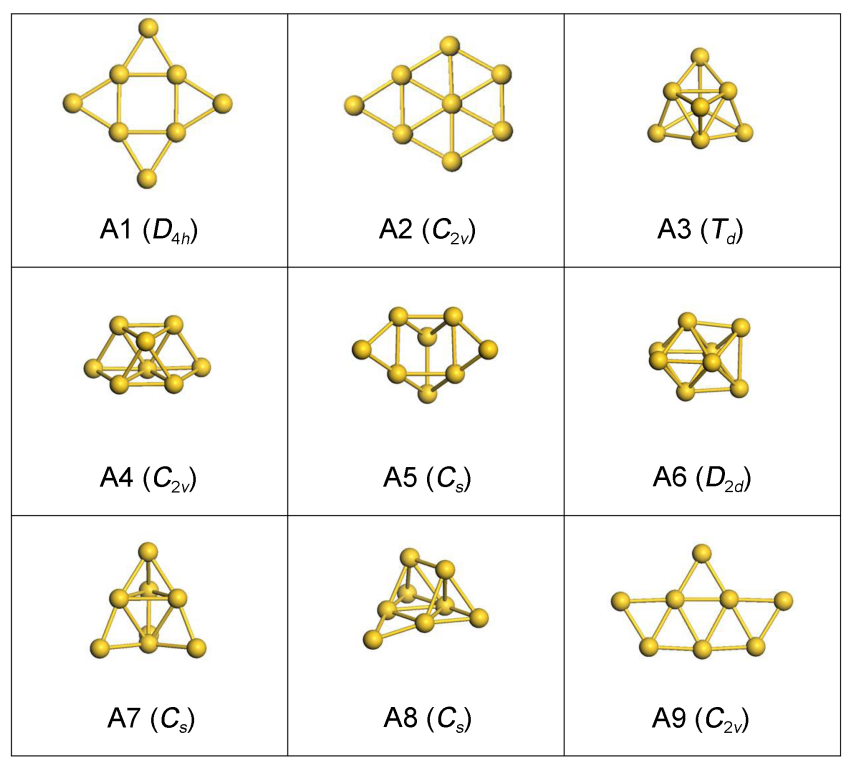

Figure 1. Optimized structures of $\mathrm{Au}_{8}$ isomers.

energies of nine isomers of $\mathrm{Au}_{8}$ (see Figure 1) computed at the $\operatorname{CCSD}(\mathrm{T}) / \mathrm{small}$ basis $+[\mathrm{MP} 2 /$ large basis $-\mathrm{MP} 2 / \mathrm{small}$ basis] level of theory. We compare the relative energies of $\mathrm{Au}_{8}$ using various DFT functionals with the more elaborate $\mathrm{ab}$ initio results in Table 2. Overall the performance of density functionals is found to be GGA hybrid GGA $>>$ LDA. The PW91 and PBE functionals, in particular, provide better performance than other functionals including the most popular B3LYP. Long range correction to the GGA functionals does not improve the performance.

The GGA and hybrid functionals correctly predict the lowest energy isomer, i.e., the planar A1 isomer. However, the LDA functionals indicate that one of the compact isomers, A4, has the lowest energy. It is worth noting that these observations for $\mathrm{Au}_{8}$ are related to the dissociation of energies of $\mathrm{Au}_{2}$. The severe overbinding $\mathrm{Au}-\mathrm{Au}$ interaction in LDA results makes the LDA calculations overestimate the stability of compact structures such as A3, A4, and A6 significantly, because much more $\mathrm{Au}-\mathrm{Au}$ bonding exists in the compact structures than in the planar isomers.

The gist of our DFT calculations is as follows:

1. Pure GGA functionals provide overall better performance than the LDA and hybrid GGA functionals for the structures and energies of gold clusters.

2. The LDA functionals can be employed for only the structural optimization of gold clusters. We suggest a GGA// LDA procedure, a GGA energy evaluation at the LDA optimized structure, for a practical method of choice. The PW91//SVWN calculations provided similar results with the PW91 results for $\mathrm{Au}_{8}$ (see Table 2).

Many theoreticians have tried to develop well-behaved density functionals for certain purposes, such as evaluation of reaction barrier height. ${ }^{23-25}$ If no panacea can be found, it is urgent to develop specific density functionals optimal for heavy element systems.

Table 2. Relative energies in $\mathrm{kcal} / \mathrm{mol}$ for $\mathrm{Au}_{8}$ isomers

\begin{tabular}{|c|c|c|c|c|c|c|c|c|c|c|}
\hline & A1 & $\mathrm{A} 2$ & A3 & A4 & A5 & A6 & A7 & A8 & A9 & $\mathrm{RMS}^{a}$ \\
\hline \multicolumn{11}{|c|}{ Local density approximation } \\
\hline SVWN & 0.0 & 2.1 & -1.4 & -3.1 & 3.2 & -1.2 & 5.5 & 2.8 & 10.5 & 5.7 \\
\hline \multicolumn{11}{|c|}{ Generalized gradient approximation } \\
\hline BLYP & 0.0 & 10.4 & 21.3 & 20.0 & 14.4 & 24.7 & 14.6 & 18.4 & 13.2 & 11.5 \\
\hline BP86 & 0.0 & 7.8 & 13.9 & 13.3 & 11.1 & 16.8 & 11.9 & 13.9 & 12.4 & 6.5 \\
\hline BPW91 & 0.0 & 8.1 & 14.4 & 14.0 & 11.6 & 17.6 & 12.3 & 14.4 & 12.4 & 7.0 \\
\hline PW91 & 0.0 & 6.9 & 11.0 & 10.4 & 9.6 & 13.5 & 10.6 & 11.9 & 12.0 & 4.5 \\
\hline PW91//SVWN & 0.0 & 7.2 & 11.8 & 11.5 & 10.1 & 14.6 & 11.0 & 12.6 & 12.0 & 5.2 \\
\hline PBE & 0.0 & 6.9 & 10.8 & 10.5 & 9.7 & 13.6 & 10.6 & 11.9 & 11.9 & 4.5 \\
\hline HCTH & 0.0 & 11.0 & 21.4 & 22.5 & 15.4 & 27.1 & 15.1 & 19.8 & 13.3 & 12.7 \\
\hline tHCTH & 0.0 & 10.8 & 21.7 & 21.6 & 15.5 & 26.3 & 15.5 & 19.8 & 13.7 & 12.4 \\
\hline LC-BPW91 & 0.0 & 12.4 & 7.4 & 10.8 & 11.2 & 15.5 & 13.2 & 13.9 & 21.7 & 5.8 \\
\hline LC-PW91 & 0.0 & 12.4 & 7.3 & 10.7 & 11.2 & 15.5 & 13.2 & 13.9 & 21.7 & 5.7 \\
\hline LC-PBE & 0.0 & 12.2 & 6.6 & 10.1 & 10.9 & 14.9 & 12.9 & 13.5 & 21.6 & 5.4 \\
\hline \multicolumn{11}{|l|}{ Hybrid GGA } \\
\hline B3LYP & 0.0 & 11.2 & 19.6 & 19.1 & 14.3 & 23.4 & 14.6 & 18.6 & 15.4 & 10.8 \\
\hline B3PW91 & 0.0 & 9.5 & 13.9 & 14.3 & 12.1 & 17.8 & 12.8 & 15.5 & 14.8 & 7.1 \\
\hline mPW1PW91 & 0.0 & 9.2 & 11.8 & 12.5 & 11.3 & 15.7 & 12.0 & 14.4 & 15.0 & 5.8 \\
\hline PBE0 & 0.0 & 8.7 & 10.0 & 10.9 & 10.4 & 13.8 & 11.2 & 13.2 & 14.8 & 4.6 \\
\hline X3LYP & 0.0 & 11.0 & 18.7 & 18.3 & 13.9 & 22.4 & 14.2 & 18.0 & 15.5 & 10.2 \\
\hline $\operatorname{CCSD}(\mathrm{T}) \mathrm{est}^{b}$ & 0.0 & 6.7 & 4.6 & 4.1 & 8.6 & 6.0 & 9.0 & 9.0 & 15.3 & 0.0 \\
\hline
\end{tabular}

${ }^{a}$ Root-mean-square deviation with respect to the $\operatorname{CCSD}(\mathrm{T})$ est results. ${ }^{b}$ Reference [10]. 
Acknowledgments. This work was supported by Korea Basic Science Institute grant (T3109A) to Y. K. H.

\section{References}

1. Pyykkö, P. Angew. Chem. Int. Ed. 2004, 43, 4412.

2. Pyykkö, P. Inorg. Chim. Acta 2005, 358, 4113.

3. Pyykkö, P. Nat. Nanotech. 2007, 2, 273.

4. Pyykkö, P. Chem. Soc. Rev. 2008, 37, 1967.

5. Haruta, M. Catal. Today 1997, 36, 153.

6. Sanchez, A.; Abbet, S.; Heiz, U.; Schneider, W.-D.; Häkkinen, H.; Barnett, R. N.; Landman, U. J. Phys. Chem. A 1999, 103, 9573.

7. Yoon, B.; Häkkinen, H.; Landman, U.; Wörz, A. S.; Antonietti, J.M.; Abbet, S.; Judai, K.; Heiz, U. Science 2005, 307, 403.

8. Lee, H. M.; Ge, M.; Sahu, B. R.; Tarakeshwar, P.; Kim, K. S. J. Phys. Chem. B 2003, 107, 9994.

9. Olson, R. M.; Varganov, S.; Gordon, M. S.; Metiu, H.; Chretien, S.; Piecuch, P.; Kowalski, K.; Kucharski, S. A.; Musial, M. J. Am. Chem. Soc. 2005, 127, 1049.

10. Han, Y.-K. J. Chem. Phys. 2006, 124, 024316.

11. Kim, K. H.; Kim, J. C.; Han, Y.-K. Bull. Kor. Chem. Soc. 2009, 30, 794.

12. Yang, X.-F.; Wang, Y.-L.; Zhao, Y.-F.; Wang, A.-Q.; Zhang, T.
Phys. Chem. Chem. Phys. 2010, 12, 3038.

13. Shao, N.; Huang, W.; Gao, Y.; Wang, L.-M.; Li, X.; Wang, L. S.; Zeng, X. C. J. Am. Chem. Soc. 2010, 132, 6596.

14. van Mourik, T. J. Chem. Theory. Comput. 2008, 4, 1610.

15. Bryantsev, V. S.; Diallo, M. S.; van Duin, A. T.; Goddard III, W. A. J. Chem. Theory. Comput. 2009, 5, 1016.

16. Shamov, G. A.; Budzelaar, P. H. M.; Schreckenbach, G. J. Chem. Theory. Comput. 2010, 6, 477.

17. Lein, M.; Krapp, A.; Frenking, G. J. Am. Chem. Soc. 2005, 127, 6290.

18. Frisch, M. J. et al. GAUSSIAN09, Revision A2; Gaussian Inc.: Pittsburgh, PA, 2009. The references are therein.

19. Stevens, W. J.; Krauss, M.; Basch, H.; Jasien, P. G. Can. J. Chem. 1992, 70, 612 .

20. Huber, K. P.; Hertzberg, G. Molecular Spectra and Molecular Structure IV. Constants of Diatomic Molecules, Van Nostrand, NY, 1979.

21. Boese, A. D.; Martin, J. M. L. J. Chem. Phys. 2004, 121, 3405.

22. Wang, N. X.; Wilson, A. K. J. Chem. Phys. 2004, 121, 7632.

23. Lynch, B. J.; Fast, P. L.; Harris, M.; Truhlar, D. G. J. Phys. Chem. A 2000, 104, 4811.

24. Song, J.-W.; Hirosawa, T.; Tsuneda, T.; Hirao, K. J. Chem. Phys. 2007, 126, 154105.

25. Zhao, Y.; Truhlar, D. G. Acc. Chem. Res. 2008, 41, 157. 